\title{
ST-segment elevation in patients with COVID-19: a systematic review
}

\author{
Carlos Diaz-Arocutipa $^{1,2,3}\left[\right.$ ] Javier Torres-Valencia ${ }^{4,5} \cdot$ Jose Saucedo-Chinchay $^{2} \cdot$ Cecilia Cuevas $^{6}$
}

Accepted: 16 February 2021 / Published online: 1 March 2021

(c) The Author(s), under exclusive licence to Springer Science+Business Media, LLC part of Springer Nature 2021

\begin{abstract}
Coronavirus disease 2019 (COVID-19) can cause a wide range of cardiovascular diseases, including ST-segment elevation myocardial infarction (STEMI) and STEMI-mimickers (such as myocarditis, Takotsubo cardiomyopathy, among others). We performed a systematic review to summarize the clinical features, management, and outcomes of patients with COVID19 who had ST-segment elevation. We searched electronic databases from inception to September 30, 2020 for studies that reported clinical data about COVID-19 patients with ST-segment elevation. Differences between patients with and without obstructive coronary artery disease (CAD) on coronary angiography were evaluated. Forty-two studies (35 case reports and seven case series) involving 161 patients were included. The mean age was $62.7 \pm 13.6$ years and $75 \%$ were men. The most frequent symptom was chest pain (78\%). Eighty-three percent of patients had obstructive CAD. Patients with non-obstructive CAD had more diffuse ST-segment elevation $(13 \%$ versus $1 \%, \mathrm{p}=0.03)$ and diffuse left ventricular wall-motion abnormality $(23 \%$ versus $3 \%, p=0.02)$ compared to obstructive CAD. In patients with previous coronary stent $(n=17)$, the $76 \%$ presented with stent thrombosis. In the majority of cases, the main reperfusion strategy was primary percutaneous coronary intervention instead of fibrinolysis. The in-hospital mortality was 30\% without difference between patients with (30\%) or without (31\%) obstructive CAD. Our data suggest that a relatively high proportion of COVID-19 patients with ST-segment elevation had non-obstructive CAD. The prognosis was poor across groups. However, our findings are based on case reports and case series that should be confirmed in future studies.
\end{abstract}

Keywords ST-segment elevation myocardial infarction · Myocardial infarction · Coronavirus disease 2019 · Systematic review

\section{Highlights}

- A relatively high proportion (17\%) of COVID-19 patients with ST-segment elevation had non-obstructive coronary

Carlos Diaz-Arocutipa

carlosdiaz013@gmail.com

1 Vicerrectorado de Investigación, Universidad San Ignacio de Loyola, Lima, Peru

2 Programa de Atencion Domiciliaria (PADOMI) - EsSalud, Lima, Peru

3 Asociación para el Desarrollo de la Investigación Estudiantil en Ciencias de la Salud (ADIECS), Lima, Peru

4 Departamento de Cardiologia, Hospital Nacional Edgardo Rebagliati Martins - EsSalud, Lima, Peru

5 Facultad de Medicina Alberto Hurtado, Universidad Peruana Cayetano Heredia, Lima, Peru

6 Instituto Nacional del Corazon (INCOR) - EsSalud, Lima, Peru artery disease (CAD) on coronary angiography.

- Patients with non-obstructive CAD had greater proportion of diffuse distribution of ST-segment elevation and left ventricular wall-motion abnormalities compared with obstructive CAD.

- A high in-hospital mortality (30\%) was reported without difference between patients with or without obstructive CAD.

\section{Introduction}

Coronavirus disease 2019 (COVID-19) was first reported in December, 2019 in Wuhan, China [1]. Since then, it has rapidly spread causing more than 32 million confirmed cases and more than 990,000 deaths across the globe [2].

The COVID-19 pandemic has overwhelmed the healthcare systems capacity from almost all countries, particularly the hospital care of emergency conditions. Several studies have shown that the number of patients with acute 
coronary syndrome admitted to the emergency departments has decreased significantly over the last months, especially after lockdown policies have been implemented [3, 4]. As a result, many of these patients may not receive optimal care, which is very important for time-dependent conditions with high morbidity and mortality such as ST-segment elevation myocardial infarction (STEMI).

It is recognized that COVID-19 is not only responsible for viral pneumonia because it can also cause a wide range of cardiovascular diseases (e.g. acute myocardial infarction, pericarditis, myocarditis, cardiac arrhythmia, heart failure, and thromboembolism), regardless of pre-existing heart conditions [5]. Thus, an adequate recognition and management of acute cardiac events such as STEMI in patients with COVID-19 are necessary. Moreover, many cardiac disorders associated with COVID-19 can manifest electrocardiographic patterns mimicking STEMI but without evidence of obstructive disease on coronary angiography [6]. Therefore, it is important to explore clinical characteristics that will differentiate patients with and without obstructive coronary artery disease (CAD) and benefit from an invasive strategy in order to reduce the exposure of catheterization laboratory personnel to patients with COVID-19. However, there is a current interest in patients with suspected or confirmed COVID-19 presenting with ST-segment elevation as there are no data-based recommendations on the management and prognosis of these high-risk patients.

Clinical reports about COVID-19 patients presenting with ST-segment elevation are being increasingly published. Also, there is a current interest in patients with both conditions because data-based recommendations to guide the appropriate diagnosis and treatment of these high-risk patients are still lacking. Likewise, considering the rapid spread of the pandemic and the second wave of COVID-19 cases that many European countries are experiencing, there is a need to rapidly assess the clinical data that has been produced. Therefore, we performed a systematic review to summarize the clinical features, management, and outcomes of COVID-19 patients with ST-segment elevation.

\section{Methods}

This review was reported according to the PRISMA (Preferred Reporting Items for Systematic Reviews and MetaAnalyses) statement [7] and was registered in the PROSPERO database (CRD42020185139).

\section{Search strategy}

We searched in the following databases: PubMed, Embase, Scopus, and Web of Science. The search was conducted from inception to May 10, 2020, with an update until
September 30, 2020. We used the following search terms with their combinations, but not limited to ST-elevation myocardial infarction, ST-segment elevation, myocardial infarction, acute coronary syndrome, coronavirus disease 2019, COVID-19, and SARS-CoV-2. The complete search strategy is available in Supplementary Table 1. There were no restrictions on language or publication date. Additionally, we conducted a hand-searching of reference lists of all included studies and relevant reviews to identify further studies. We also searched the articles citing the selected studies (through Google Scholar) and the preprint servers medRxiv (https://www.medrxiv.org/) and SSRN (https:// www.ssrn.com/) for additional studies.

\section{Eligibility criteria}

The inclusion criteria were the following: (i) studies that included adult patients ( $\geq 18$ years of age) diagnosed with COVID-19 by reverse transcription-polymerase chain reaction (RT-PCR); (ii) studies that reported patients with ST-segment elevation on electrocardiogram (ECG) on admission or during hospitalization and reporting data on cardiac catheterization or coronary computed tomography angiography; (iii) studies that described at least one of the following information: clinical features, management, or outcomes of COVID-19 patients with ST-segment elevation; (iv) case reports, case series, cross-sectional, case-control, and cohort studies; and (v) original articles and research letters. We excluded animal studies, abstracts, editorials, commentaries, systematic reviews, and narrative reviews.

\section{Study selection}

Two review authors (CDA and JTV) downloaded all articles from electronic search to EndNote X8 software and duplicate records were removed. All unique articles were uploaded to Rayyan QCRI (https://rayyan.qcri.org/) for the selection process. Titles and abstracts were independently screened by two review authors (CDA and JTV) to identify relevant studies. Likewise, two review authors (CDA and JTV) independently examined the full-text of each eligible study and registered reasons for the exclusion. Any disagreement on title/abstract and full-text selection was resolved by consensus.

\section{Data extraction}

We extracted the following data: first author name, publication year, country, study design, sample size, age, sex, comorbidities, clinical features, electrocardiographic findings, laboratory, echocardiographic findings, reperfusion strategy, coronary interventions, and outcomes. If additional 
data was needed, we contacted the corresponding author through email.

\section{Methodological quality assessment}

We used a modified version of the Newcastle-Ottawa Scale (NOS) to assess the methodological quality of case reports and case series [8]. This tool has been applied in previous systematic reviews with consistency among reviewers [9-11]. Each study was judged on five questions with a binary response (yes or no). The quality was considered good if all five criteria were fulfilled, moderate if four were fulfilled, and low if three or less were fulfilled. Two review authors (CDA and CCD) independently assessed the quality for each study and any disagreement was resolved by consensus.

\section{Statistical analysis}

For univariate analysis, frequencies and proportions were used to summarize categorical variables. Whereas, means \pm standard deviations or median (interquartile range) were used for continuous variables. For bivariate analysis, we compared patient characteristics, clinical features, diagnostic methods, management, and outcomes between patients with and without obstructive coronary artery disease (CAD). Obstructive CAD was defined as $\geq 50 \%$ stenosis and/or the presence of thrombus on any epicardial coronary artery. Pearson's chi-squared test or Fisher's exact test was used for categorical variables and unpaired Student's t-test for continuous variables. All statistical analyses were performed using the statistical software R 3.6.3 (www.r-project.org). A two-tailed p $<0.05$ was considered as statistically significant.

\section{Results}

\section{Study selection}

Our electronic search retrieved 251 articles. After the removal of duplicates, 161 articles were reviewed based on the title and abstract, and of those, 105 articles were excluded. After full-text assessment of 56 remaining articles, 14 were excluded: two studies without STEMI patients, four studies without data on coronary angiography, one narrative review, and seven studies with no
Fig. 1 Flow diagram of study selection

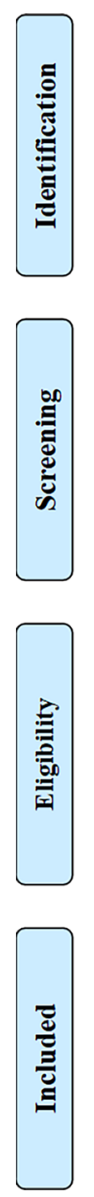

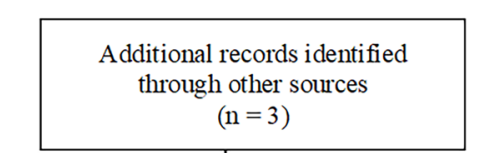

$(\mathrm{n}=248)$
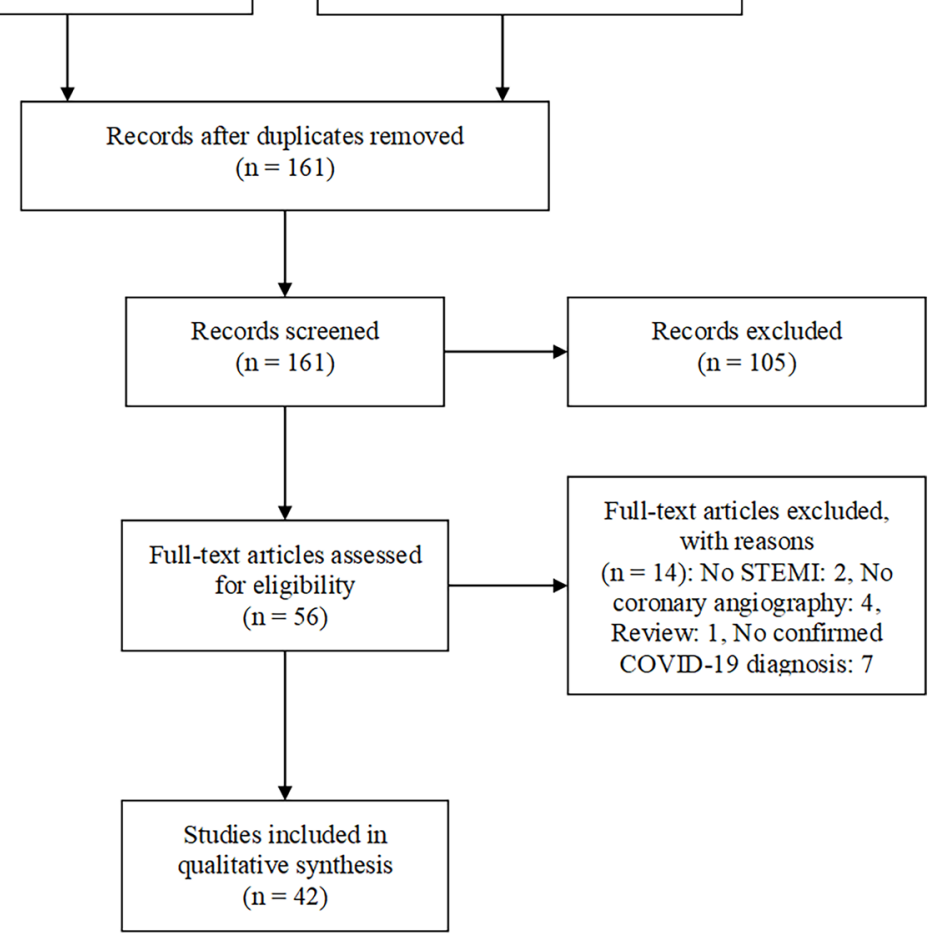
confirmed COVID-19 diagnosis. Finally, 42 studies were selected for analysis (Fig. 1).

\section{Methodological quality assessment}

The quality assessment of the case series was scored as good for four studies, moderate for two studies, and low for one study (Supplementary Table 2). Twenty-six case reports were rated as good quality and eight case reports as moderate quality (Supplementary Table 2). All studies were included in the analysis regardless of their methodological quality.

\section{Study characteristics and demographics}

The principal characteristics of the 42 included studies [S1-S42] (seven case series and 35 case reports) are summarized in Supplementary Table 3. We found a total of 161 adult patients diagnosed with ST-segment elevation and COVID-19 (Table 1). The mean age was $62.7 \pm 13.6$ years and $75 \%$ of patients were men. The proportion of men was significantly higher in patients with obstructive coronary artery disease (CAD) compared to non-obstructive CAD (79\% versus 57\%). Most cases were from Italy (27\%).

\section{Comorbidities and clinical features}

Hypertension was the most common comorbidity both in patients with (59\%) and without (70\%) CAD (Table 1). Previous $\mathrm{CAD}$ was reported in $20 \%$ of patients and there was no significant difference between patients with obstructive and non-obstructive CAD. Other associated conditions such as diabetes (37\%), dyslipidemia (42\%), and chronic kidney disease (9\%) were also reported. Among symptoms at presentation, the most frequent was chest pain (78\%).

\section{Diagnostic methods}

Overall, the ST-segment elevation was similarly localized both in the septal/anterior (48\%) and inferior/lateral/posterior $(46 \%)$ walls of the left ventricle (Table 1$)$. The proportion of septal/anterior distribution was higher in patients with obstructive CAD. In contrast, a diffuse ST-segment elevation was more significantly found in patients with non-obstructive CAD. In 107 of 133 patients (80\%), STsegment elevation was reported on hospital admission, while in the rest, occurred after admission. The mean left ventricular ejection fraction was $38.9 \pm 12.5 \%$ with no difference between both groups (Table 1). Patients with obstructive CAD presented more regional wall-motion abnormalities
(97\%) than patients without obstructive CAD (63\%). The normal left ventricular wall-motion was present more frequently in patients with non-obstructive CAD (14\%).

\section{Management and outcomes}

Invasive coronary angiography was performed in $99 \%$ of patients. Only one patient was evaluated with coronary computed tomography angiography [S13]. Obstructive CAD was found in 133 of 161 patients (83\%) (Table 1). In patients with non-obstructive CAD, the most common diagnoses were non-coronary myocardial injury $[S 1, S 2, S 20$, $\mathrm{S} 27]$ ( $\mathrm{n}=16$ patients), pericarditis/myocarditis [S4, $\mathrm{S} 9$, $\mathrm{S} 13, \mathrm{~S} 21, \mathrm{~S} 28]$ ( $\mathrm{n}=7$ patients), and Takotsubo syndrome [S3, S30, S34] ( $n=5$ patients). Patients were classified as "non-coronary myocardial injury" when coronary angiography reported non-obstructive CAD. A total of 17 patients reported a previously implanted coronary stent $[\mathrm{S} 6, \mathrm{~S} 7, \mathrm{~S} 8$, S10, S11, S17, S25, S27, S39, S40]. In 13 of them (74\%), stent thrombosis was found on coronary angiography [S6, S10, S11, S17]. Only six cases had information about timing of stent thrombosis: one acute [S10], two late [S7, S39], and three very late [S8, S17, S40]. The left anterior descending artery was the most frequent culprit lesion (45 of 76 patients) in STEMI patients.

Primary percutaneous coronary intervention (PCI) was the main reperfusion strategy in COVID-19 patients with ST-segment elevation (93\%) (Table 1). In contrast, fibrinolysis was performed in only 11 patients. Complete information about the success of fibrinolytic therapy was not available in nearly all cases. No difference in reperfusion strategy was reported between patients with and without obstructive CAD. Stent implantation and aspiration thrombectomy were performed in $91 \%$ and $12 \%$ of patients with obstructive CAD, respectively (Table 1). Overall, 46 of 151 patients (30\%) died during hospitalization with no difference between both groups. Six patients remain hospitalized during the study period (Table 1).

\section{Discussion}

In our systematic review of COVID-19 patients with STsegment elevation, $83 \%$ of patients had obstructive CAD on coronary angiography. The ST-segment elevation and left ventricular wall-motion abnormalities showed a regional distribution in the majority of cases. In nearly all patients, the main reperfusion strategy was primary PCI with stent implantation. Overall, the in-hospital mortality was $30 \%$ without difference between patients with obstructive and non-obstructive CAD.

We found that most of the patients presented with STsegment elevation on ECG at hospital admission, while only 
Table 1 Characteristics of COVID-19 patients with ST-segment elevation

\begin{tabular}{|c|c|c|c|c|}
\hline Characteristics & All patients $(n=161)$ & Obstructive CAD $(\mathrm{n}=133)$ & Non-obstructive CAD $(\mathrm{n}=28)$ & p-value \\
\hline \multicolumn{5}{|l|}{ Country (\%) } \\
\hline Italy & $43 / 161(27 \%)$ & $27 / 133(20 \%)$ & $16 / 28(57 \%)$ & $<0.01 *$ \\
\hline UK & $41 / 161(25 \%)$ & $41 / 133(31 \%)$ & $0 / 28(0 \%)$ & - \\
\hline Lithuania, Italy, Spain, Iraq & 27/161 (17\%) & 25/133 (19\%) & $2 / 28(7 \%)$ & $0.17 * *$ \\
\hline France & $12 / 161(7 \%)$ & $12 / 133(9 \%)$ & $0 / 28(0 \%)$ & - \\
\hline USA & $21 / 161(13 \%)$ & $14 / 133(10 \%)$ & $7 / 28(25 \%)$ & $0.06^{* *}$ \\
\hline Others & $17 / 161(11 \%)$ & $14 / 133(11 \%)$ & $3 / 28(11 \%)$ & $1 * *$ \\
\hline Age (years), mean \pm SD & $62.7 \pm 13.6, n=65$ & $63.2 \pm 13.5, n=44$ & $61.8 \pm 14.2, \mathrm{n}=21$ & $0.69 * * *$ \\
\hline Male $(\%)$ & $86 / 115(75 \%)$ & 74/94 (79\%) & $12 / 21(57 \%)$ & $0.03^{*}$ \\
\hline \multicolumn{5}{|l|}{ Comorbidities (\%) } \\
\hline Hypertension & $68 / 112(61 \%)$ & $54 / 92(59 \%)$ & $14 / 20(70 \%)$ & $0.35^{*}$ \\
\hline Diabetes & $42 / 112(37 \%)$ & $39 / 92(42 \%)$ & $3 / 20(15 \%)$ & $0.02 *$ \\
\hline CAD & $23 / 112(20 \%)$ & $20 / 92(22 \%)$ & $3 / 20(15 \%)$ & $0.76^{* *}$ \\
\hline Dyslipidemia & $47 / 112(42 \%)$ & $42 / 92(46 \%)$ & $5 / 20(25 \%)$ & $0.09 *$ \\
\hline CKD & $10 / 112(9 \%)$ & $4 / 92(4 \%)$ & $6 / 20(30 \%)$ & $<0.01 * *$ \\
\hline \multicolumn{5}{|l|}{ Symptoms (\%) } \\
\hline Chest pain & $50 / 64(78 \%)$ & $36 / 43(84 \%)$ & $14 / 21(67 \%)$ & $0.19 * *$ \\
\hline Dyspnea & $36 / 64(56 \%)$ & $22 / 43(51 \%)$ & $14 / 21(67 \%)$ & $0.24 *$ \\
\hline \multicolumn{5}{|c|}{ Localization of ST-segment elevation (\%) } \\
\hline Septal/anterior & $53 / 111(48 \%)$ & $47 / 87(54 \%)$ & $6 / 24(25 \%)$ & $0.01 *$ \\
\hline Inferior/lateral/posterior & $51 / 111(46 \%)$ & $38 / 87(44 \%)$ & $13 / 24(54 \%)$ & $0.36^{*}$ \\
\hline Diffuse & $4 / 111(3 \%)$ & $1 / 87(1 \%)$ & $3 / 24(13 \%)$ & $0.03 * *$ \\
\hline New LBBB & $3 / 111(3 \%)$ & $1 / 87(1 \%)$ & $2 / 24(8 \%)$ & $0.12 * *$ \\
\hline \multicolumn{5}{|l|}{ Echocardiogram (\%) } \\
\hline $\mathrm{LVEF}$, mean $\pm \mathrm{SD}$ & $38.9 \pm 12.5, \mathrm{n}=55$ & $37.5 \pm 12.6, n=36$ & $41.8 \pm 12.3, \mathrm{n}=19$ & $0.23 * * *$ \\
\hline Regional wall-motion abnormality & $48 / 57(84 \%)$ & $34 / 35(97 \%)$ & $14 / 22(63 \%)$ & $<0.01 * *$ \\
\hline Diffuse wall-motion abnormality & $6 / 57(11 \%)$ & $1 / 35(3 \%)$ & $5 / 22(23 \%)$ & $0.02 * *$ \\
\hline No wall-motion abnormality & $3 / 57(5 \%)$ & $0 / 35(0 \%)$ & $3 / 22(14 \%)$ & - \\
\hline \multicolumn{5}{|l|}{ Type of reperfusion strategy (\%) } \\
\hline Fibrinolysis & $11 / 161(7 \%)$ & $10 / 133(8 \%)$ & $1 / 28(4 \%)$ & $1 * *$ \\
\hline Primary PCI & $150 / 161(93 \%)$ & $123 / 133(92 \%)$ & $27 / 28(96 \%)$ & $1 * *$ \\
\hline \multicolumn{5}{|l|}{ Type of coronary intervention (\%) } \\
\hline Stent implantation & $121 / 161(75 \%)$ & $121 / 133(91 \%)$ & $0 / 28(0 \%)$ & - \\
\hline Aspiration thrombectomy & $16 / 161(10 \%)$ & $16 / 133(12 \%)$ & $0 / 28(0 \%)$ & - \\
\hline Only balloon angioplasty & $3 / 161(2 \%)$ & $3 / 133(2 \%)$ & $0 / 28(0 \%)$ & - \\
\hline None & $34 / 161(21 \%)$ & $6 / 133(4 \%)$ & $28 / 28(100 \%)$ & - \\
\hline \multicolumn{5}{|l|}{ Outcomes $(\%)$} \\
\hline Hospitalized & $6 / 151(4 \%)$ & $4 / 125(3 \%)$ & $2 / 26(8 \%)$ & $0.27 * *$ \\
\hline Discharged & $99 / 151(66 \%)$ & $83 / 125(67 \%)$ & $16 / 26(61 \%)$ & $0.63 *$ \\
\hline Dead & $46 / 151(30 \%)$ & $38 / 125(30 \%)$ & $8 / 26(31 \%)$ & $0.97 *$ \\
\hline
\end{tabular}

$C A D$ coronary artery disease, $C K D$ chronic kidney disease, $C T$ computerized tomography, $L V E F$ left ventricular ejection fraction, $L B B B$ left bundle branch block, $S D$ standard deviation, $I Q R$ interquartile range, USA United States of America, UK United Kingdom, $R T-P C R$ reverse transcription-polymerase chain reaction, $P C I$ percutaneous coronary intervention

*Chi-square test

**Fisher's exact test

***t-student test 
$20 \%$ of patients developed this electrocardiographic pattern during hospitalization. Due to COVID-19 status is unknown for many individuals at the time of cardiac catheterization laboratory activation, it is necessary an appropriate and timely selection of patients for invasive coronary angiography to reduce health staff exposure and optimize the personal protective equipment utilization [6]. Current consensus guidelines for STEMI management during the COVID-19 pandemic from the American College of Cardiology, Society for Cardiovascular Angiography and Interventions, European Society of Cardiology, and European Association of Percutaneous Cardiovascular Interventions recommend that primary PCI strategy should remain the standard of care for patients with persistent ST-segment elevation less than $12 \mathrm{~h}$ and suspected or confirmed COVID-19 [6, 12, 13]. Alternatively, a pharmacoinvasive approach may be considered if a primary PCI is not feasible. Our review showed that the principal reperfusion strategy used in STEMI patients with COVID-19 was primary PCI and only $7 \%$ of patients were treated with fibrinolytic therapy. These findings are in accordance with current guidelines; however, more studies with large samples and higher follow-up are needed to support these recommendations.

There are several reports on STEMI mimics (e.g. pericarditis/myocarditis, Takotsubo syndrome, microvascular thrombosis, and thromboembolism) known to be associated with COVID-19 [14]. A pre-pandemic prospective cohort of 489 patients who presented to the emergency department with ST-segment elevation on ECG found that $11 \%$ of cases had no culprit lesion on coronary angiography [15]. The most common etiologies were early repolarization, pericarditis/myocarditis, and Takotsubo syndrome [15]. In comparison, our study showed a higher proportion (19\%) of COVID-19 patients presenting with ST-segment elevation that had non-obstructive CAD. The largest reported proportion of non-coronary lesion on coronary angiography in our review was $39 \%$ in a case series ( $n=28$ patients) from Italy [S1]. Similar to the previous study, pericarditis/myocarditis and Takotsubo syndrome were also the most frequently reported diagnoses. In addition, a German cohort of 100 patients recently recovered from COVID-19 reported that $60 \%$ of patients had ongoing myocardial inflammation on cardiovascular magnetic resonance, showing the frequent non-coronary involvement of COVID-19 [16]. Overall, these data highlight the need that other diagnoses than STEMI such as myocarditis should be considered during the evaluation of COVID-19 patients with ST-segment elevation.

Interestingly, we found that patients without obstructive CAD on coronary angiography had significantly more diffuse ST-segment elevation on ECG compared to patients with obstructive CAD. This relevant electrocardiographic difference could be used to identify patients which are unlikely to benefit from an invasive approach. However, this finding should be confirmed in future studies.

Evidence of altered coagulation tests appeared in early reports from Wuhan $[17,18]$. In addition, several venous and arterial thrombotic events have been reported in COVID19 patients [19]. A recent analysis of 115 patients presenting with STEMI showed that the COVID-19 group had a higher coronary thrombotic burden compared to the nonCOVID-19 group [S6]. Although it was not possible to assess the thrombotic burden in all patients due to lack of uniformed reporting, we found a surprisingly high proportion $(74 \%)$ of stent thrombosis in patients with previous coronary stent. The intense pro-inflammatory response induced by COVID-19 appears to be responsible for the hypercoagulable and pro-thrombotic status which is probably triggered by endothelial dysfunction, hypoxia, and abnormal platelet activity [20]. Moreover, it has been suggested that COVID19 patients may have at increased risk of coronary plaque rupture, occlusive thrombus formation, and STEMI [14]. Further research is required to clarify the pathophysiological mechanisms of coronary thrombosis in COVID-19 patients.

A main concern in the current COVID-19 pandemic is the mortality rate of infected patients. The current estimate of global mortality in COVID-19 patients is 3.4\% based on data from the World Health Organization [2]. In our review, inhospital mortality of STEMI patients with COVID-19 was disproportionally high (30\%), even exceeding the reported in-hospital mortality of unselected STEMI patients which ranged from 3 to $12 \%$ across 11 European countries [21].

To the best of our knowledge, this is the first systematic review that summarized the current clinical information on ST-segment elevation in COVID-19 patients. We conducted a comprehensive search that included all published data on this topic. However, our study has some limitations. First, given that included studies were case reports and case series, causality cannot be concluded due to the methodological limitations of these designs. Also, publication and selection bias would be probably present, since only cases with unusual features and worse outcomes may have been published affecting the global findings of the study. Our analysis showed no difference in mortality between both groups, although patients from case series had more obstructive CAD on coronary angiography. Second, information on individual patients was absent for many variables in four of seven case series which limits the sample size for comparisons. Thus, adjustment for confounders was not possible for all associations. Finally, our study was composed of only 161 patients, which is a relatively small sample, precluding the generalizability of results. 


\section{Conclusion}

Our systematic review shows that obstructive CAD was found in most of the COVID-19 patients who had ST-segment elevation. However, a relatively high proportion of non-obstructive CAD was present. The main reperfusion strategy used was primary PCI rather than fibrinolysis. In patients with previous coronary stent, a disproportionally high proportion of stent thrombosis was reported. The inhospital mortality was high without difference between patients with obstructive and non-obstructive CAD. Although we have summarized all relevant data from published studies, this information came only from case reports and case series. Therefore, larger and prospective studies are still required to provide both short-term and long-term clinical data for the optimal care of these high-risk patients.

Supplementary Information The online version contains supplementary material available at https://doi.org/10.1007/s11239-021-02411-9.

Author contributions All authors contributed to the study conception and design. Articles selection, data extraction, and data analysis were performed by CDA and JTV. The first draft of the manuscript was written by CDA and all authors commented on previous versions of the manuscript. All authors read and approved the final manuscript.

\section{Funding None.}

\section{Declarations}

Conflict of interest The authors declare that they have no conflict of interest.

\section{References}

1. Zhou P, Yang XL, Wang XG, Hu B, Zhang L, Zhang W, Si HR, Zhu Y, Li B, Huang CL, Chen HD, Chen J, Luo Y, Guo H, Jiang RD, Liu MQ, Chen Y, Shen XR, Wang X, Zheng XS, Zhao K, Chen QJ, Deng F, Liu LL, Yan B, Zhan FX, Wang YY, Xiao GF, Shi ZL (2020) A pneumonia outbreak associated with a new coronavirus of probable bat origin. Nature 579(7798):270-273. https://doi.org/10.1038/s41586-020-2012-7

2. WHO Coronavirus Disease (COVID-19) Dashboard. https://covid 19.who.int/. accessed 30 Sept 2020

3. Mafham MM, Spata E, Goldacre R, Gair D, Curnow P, Bray M, Hollings S, Roebuck C, Gale CP, Mamas MA, Deanfield JE, de Belder MA, Luescher TF, Denwood T, Landray MJ, Emberson JR, Collins R, Morris EJA, Casadei B, Baigent C (2020) COVID19 pandemic and admission rates for and management of acute coronary syndromes in England. Lancet S0140-6736(20):3135631358. https://doi.org/10.1016/s0140-6736(20)31356-8

4. Mountantonakis SE, Saleh M, Coleman K, Kuvin J, Singh V, Jauhar R, Ong L, Qiu M, Epstein LM (2020) Out-of-hospital Cardiac Arrest and Acute Coronary Syndrome Hospitalizations during the COVID-19 Surge. J Am Coll Cardiol S0735-1097(20):3596435967. https://doi.org/10.1016/j.jacc.2020.07.021

5. Nishiga M, Wang DW, Han Y, Lewis DB, Wu JC (2020) COVID19 and cardiovascular disease: from basic mechanisms to clinical perspectives. Nat Rev Cardiol. https://doi.org/10.1038/s4156 9-020-0413-9

6. Mahmud E, Dauerman HL, Welt FGP, Messenger JC, Rao SV, Grines C, Mattu A, Kirtane AJ, Jauhar R, Meraj P, Rokos IC, Rumsfeld JS, Henry TD (2020) Management of acute myocardial infarction during the COVID-19 pandemic: A Consensus Statement from the Society for Cardiovascular Angiography and Interventions (SCAI), the American College of Cardiology (ACC), and the American College of Emergency Physicians (ACEP). Catheter Cardiovasc Interv. https://doi.org/10.1002/ ccd.28946.10.1002/ccd.28946

7. Moher D, Liberati A, Tetzlaff J, Altman DG (2009) Preferred reporting items for systematic reviews and meta-analyses: the PRISMA statement. BMJ 339:b2535. https://doi.org/10.1136/ bmj.b2535

8. Murad MH, Sultan S, Haffar S, Bazerbachi F (2018) Methodological quality and synthesis of case series and case reports. BMJ Evid Based Med 23(2):60-63. https://doi.org/10.1136/ bmjebm-2017-110853

9. Bazerbachi F, Haffar S, Sugihara T, Mounajjed TM, Takahashi N, Murad MH, Abu Dayyeh BK (2018) Peribiliary cysts: a systematic review and proposal of a classification framework. BMJ Open Gastroenterol 5(1):e000204. https://doi.org/10.1136/ bmjgast-2018-000204

10. Haffar S, Kaur RJ, Wang Z, Prokop LJ, Murad MH, Bazerbachi F (2018) Acute liver failure caused by hepatitis E virus genotype 3 and 4: a systematic review and pooled analysis. Liver Int 38(11):1965-1973. https://doi.org/10.1111/liv.13861

11. Montagnon CM, Fracica EA, Patel AA, Camilleri MJ, Murad MH, Dingli D, Wetter DA, Tolkachjov SN (2020) Pyoderma gangrenosum in hematologic malignancies: a systematic review. J Am Acad Dermatol 82(6):1346-1359. https://doi. org/10.1016/j.jaad.2019.09.032

12. ESC Guidance for the Diagnosis and Management of CV Disease during the COVID-19 Pandemic. https://www.escardio.org/ Education/COVID-19-and-Cardiology/ESC-COVID-19-Guida nce\#p09. Accessed Aug 22020.

13. Chieffo A, Stefanini GG, Price S, Barbato E, Tarantini G, Karam N, Moreno R, Buchanan GL, Gilard M, Halvorsen S, Huber K, James S, Neumann FJ, Möllmann H, Roffi M, Tavazzi G, Ferré JM, Windecker S, Dudek D, Baumbach A (2020) EAPCI Position Statement on Invasive Management of Acute Coronary Syndromes during the COVID-19 pandemic. EuroIntervention 16(3):233-246. https://doi.org/10.4244/eijy20m05_01

14. Azevedo RB, Botelho BG, Hollanda JVG, Ferreira LVL, Junqueira de Andrade LZ, Oei S, Mello TS, Muxfeldt ES (2020) COVID-19 and the cardiovascular system: a comprehensive review. J Hum Hypertens. https://doi.org/10.1038/s4137 1-020-0387-4

15. Nfor T, Kostopoulos L, Hashim H, Jan MF, Gupta A, Bajwa T, Allaqaband S (2012) Identifying false-positive ST-elevation myocardial infarction in emergency department patients. J Emerg Med 43(4):561-567. https://doi.org/10.1016/j.jemer med.2011.09.027

16. Puntmann VO, Carerj ML, Wieters I, Fahim M, Arendt C, Hoffmann J, Shchendrygina A, Escher F, Vasa-Nicotera M, Zeiher AM, Vehreschild M, Nagel E (2020) Outcomes of cardiovascular magnetic resonance imaging in patients recently recovered from coronavirus disease 2019 (COVID-19). JAMA Cardiol. https:// doi.org/10.1001/jamacardio.2020.3557

17. Chen N, Zhou M, Dong X, Qu J, Gong F, Han Y, Qiu Y, Wang J, Liu Y, Wei Y, Xia J, Yu T, Zhang X, Zhang L (2020) Epidemiological and clinical characteristics of 99 cases of 2019 novel coronavirus pneumonia in Wuhan, China: a descriptive study. Lancet 395(10223):507-513. https://doi.org/10.1016/s0140 $-6736(20) 30211-7$ 
18. Wang D, Hu B, Hu C, Zhu F, Liu X, Zhang J, Wang B, Xiang H, Cheng Z, Xiong Y, Zhao Y, Li Y, Wang X, Peng Z (2020) Clinical characteristics of 138 hospitalized patients with 2019 novel coronavirus-infected pneumonia in Wuhan, China. JAMA 323(11):1061-1069. https://doi.org/10.1001/jama.2020.1585

19. Bilaloglu S, Aphinyanaphongs $\mathrm{Y}$, Jones S, Iturrate E, Hochman J, Berger JS (2020) Thrombosis in hospitalized patients with COVID-19 in a New York City Health System. JAMA. https:// doi.org/10.1001/jama.2020.13372

20. Connors JM, Levy JH (2020) COVID-19 and its implications for thrombosis and anticoagulation. Blood 135(23):2033-2040. https ://doi.org/10.1182/blood.2020006000

21. Kristensen SD, Laut KG, Fajadet J, Kaifoszova Z, Kala P, Di Mario C, Wijns W, Clemmensen P, Agladze V, Antoniades L,
Alhabib KF, De Boer MJ, Claeys MJ, Deleanu D, Dudek D, Erglis A, Gilard M, Goktekin O, Guagliumi G, Gudnason T, Hansen KW, Huber K, James S, Janota T, Jennings S, Kajander O, Kanakakis J, Karamfiloff KK, Kedev S, Kornowski R, Ludman PF, Merkely B, Milicic D, Najafov R, Nicolini FA, Noč M, Ostojic M, Pereira H, Radovanovic D, Sabaté M, Sobhy M, Sokolov M, Studencan M, Terzic I, Wahler S, Widimsky P (2014) Reperfusion therapy for ST elevation acute myocardial infarction 2010/2011: current status in 37 ESC countries. Eur Heart J 35(29):1957-1970. https://doi.org/10.1093/eurheartj/eht529

Publisher's Note Springer Nature remains neutral with regard to jurisdictional claims in published maps and institutional affiliations. 